\title{
Are we providing a good service for people with chronic schizophrenia?
}

John Wing

The first target of the government's mental Health of the Nation strategy is to improve the health and social functioning of mentally ill people. Most health service professionals would agree with the implication that there is substantial room for improvement. It is as important to be aware of the extent to which a service is failing as it is to know that needs are being met.

The obvious answer to the question in the title is to ask the patients themselves, and also the informal carers (there is no consensus on preferred terminology, the terms 'carer' and 'patient' are used for convenience). Such questions need to be phrased on the basis of a good understanding of two different, but overlapping, perspectives - one 'schizophrenia from within', the other 'schizophrenia at home' - together with a readiness to adapt the formulation to the individual circumstances that make each such discussion unique. In parallel with many other distressing medical conditions, clinicians may find that achieving such dual understanding takes time and can be difficult, but it is possible and well worth the effort. The first part of the paper deals with this.

The second part of the paper is concerned with a more technical kind of answer, which should inform the first. The quality of personal clinical practice should be routinely compared against professional consensus guidelines, within the context of good service information, as part of the clinical audit and needs assessment cycle. This includes diagnosis, problem recognition, prescription of treatments, choice of staff, allocation of care setting and clinical recording. A further level for appraisal, that of service provision and quality, must also be considered because, although not always within the immediate power of consultants to influence, it certainly bears on their ability to provide a good service for patients.
Both parts of the paper deal with factors that are not under the control of consultants as well as factors that are. All clinicians should know about both.

\section{Consulting patients and carers}

Formulating the problems posed to a particular person who is experiencing schizophrenia requires recognition of those that arise specifically from the phenomena and those that are part of the broader personal and social environment. The impact of the familiar textbook symptoms of the first rank and the 'negative' impairments can be appreciated with an effort of imagination. A booklet published by the National Schizophrenia Fellowship (NSF) provides seven unvarnished personal accounts of schizophrenia from within, which can help other people comprehend something of the fearful experiences involved. The authors describe the frustration of long-term handicap or frequent relapse, and of knowing that the problems are 'invisible' so that the sympathy extended to people with more evident disabilities is withheld. They make useful suggestions as to how other people can react most helpfully and they emphasise selfhelp. The essays illustrate the fact that some of those afflicted can work out for themselves ways of living with their own specific problems that are now codified under the name of cognitive therapy.

Together with a survey of relatives' experiences published by the NSF, a substantial literature accumulated over the past 20 years (Creer \& Wing, 1974; Wing, 1975; Torry, 1995), and recently produced NSF video-tapes, there is plenty of material to add another, more personal, perspective to the phenomenology that has accumulated during the past century and a half.

John Wing is Emeritus Professor of Social Psychiatry, University of London. He was Director of the MRC Social Psychiatry Unit until September 1989 and Director of the Royal College of Psychiatrists' Research Unit (where he still works) until September 1994. 


\section{Disability may be long-term but it is not necessarily immutable}

There is some dispute as to where the distinction between positive and negative phenomena should be drawn, but a reasonable compromise is to separate blunting of affect, psychomotor poverty and social withdrawal on the one hand from first rank symptoms and accompanying manifestations on the other. This leaves incoherence of speech (and therefore of thought) for allocation as a possible third group, although it is generally associated with social withdrawal as well. All three can be present within the same episode.

There are practical as well as theoretical reasons for making such distinctions. People with negative symptoms are particularly vulnerable to poverty of the social environment, which can lead to an increase in slowness, social withdrawal and apathy. Such conditions may be found in hospital wards, hostels, lodgings, day centres, at home or on the streets. Encouragement to be active up to the level of the individual's ability is therapeutic. Specific provision is therefore required in every district for a range of graded activities in workshops and recreation centres, supervised by qualified staff familiar with the specific as well as the non-specific problems of schizophrenia. If these are not available or not utilised the vulnerable patient is not receiving the most appropriate care.

A further problem is that negative symptoms impair the ability to communicate with other people, both through poverty or incoherence of speech and through body language. This is a double disability, because people who might potentially be supportive (including relatives, friends and professionals) may assume from what appears to be an unfriendly and unresponsive manner or actual avoidance of contact that attempts at communication are fruitless, so that fewer and fewer attempts are made to reach out and help. This process can become self-perpetuating as the patient finds it easier to give up trying.

Positive symptoms entail communication and behavioural problems of a more obvious kind and also a different kind of vulnerability to the environment. There are promising techniques for helping to ameliorate even long-standing symptoms (Sellwood et al, 1994). But events that most people take in their stride may lead to further breakdown. Unduly vigorous attempts at rehabilitation without preparation can increase the pressure too much. Emotional pressure from professionals or relatives can have the same effect.

Patients with both kinds of vulnerability therefore have to face two kinds of danger. On the one hand, too little pressure encourages social withdrawal, slowness and apathy. On the other, too much can lead to a recrudescence of the acute schizophrenic experiences. People with schizophrenia seem to have a sharpened Yerkes-Dodson curve. It was demonstrated long ago that the milieu of a sheltered workshop in which patients were given emotionally-neutral encouragement to perform up to their optimal level of ability was a model for the creation of care environments.

Relatives also have to live and try to come to terms with both kinds of problem. One mother said her son spent most of his time closeted in his room, only coming out at night when everyone was in bed. Usually he was talking to himself or moving about but every few weeks there would be complete silence. "After that has been going on for a day or two I sometimes wonder whether he is dead." Dealing with delusions that are focused on the relative is particularly difficult. Emergency admission to hospital is traumatic for all concerned. Understanding the ins and outs of the Mental Health Act and Supervised Registers is beyond some relatives. Other difficulties involve coping with irritable or aggressive patients, refusal to accept medication, use of hard and soft drugs, nonavailability of day and residential respite services, and problems in obtaining welfare benefits when patients refuse to collect them.

Such problems cause divisions within the family which can lead to break-up. Getting useful advice about how to react to such problems can be very difficult. Too often, professionals respond to questions about what to do about difficult behaviour with: "What do you do?", followed by no constructive expert advice of their own. Relatives in a position to help need information. In such cases, consultants should be wary of withholding it if the patient refuses permission. With reference to the poor uptake of benefits, Slade et al (1995) recommend as follows:

"Further training for staff who routinely deal with mentally ill people could be complemented by the use of computer evaluation tools. If community care is to be a reality, then changes will be needed to ensure that vulnerable people are given the protection that welfare benefits can provide."

The authors point out that staff ignorance is not confined to health personnel; it applies also to social workers. All the other problems listed above raise similar issues of training and the availability of useful knowledge. The after-discharge (including Section 117) form recommended by the Department of Health for completion by all relevant authorities and organisations provides a similar opportunity. Slade and colleagues point out that help can be obtained by the provision of, and training for 
Box 1. Clinical appraisal against guidelines. Consensus guidelines are needed (some are already available) for each level of clinical appraisal:

Current profile of health and social problems (possible needs)

ICD-10 diagnosis

Symptomatic and behavioural profile Activities of daily living, social functioning, autonomy

Interventions appropriate to each problem identified

Staff well qualified to provide the interventions

Supportive care settings (residential, domestic, occupational, recreational, etc)

familiarity with, simple computer aids. This subject is considered in more detail in a later section.

Understanding the impact of the symptoms on the patient can make all the difference between acceptance and rejection by the relative. If it is recognised that negative symptoms such as slowness and communication difficulties are not due to laziness or unfriendliness, that hallucinations are not imaginary, and that delusions are not deliberate lies, the problem of coping is radically changed. Many relatives do discover for themselves how to limit disability and distress and provide the patient with a supportive home. But many others need help to gain this understanding and some may need confirmation that it would be better for the patient to reduce contact. Kuipers (1994) provides a useful summary of the literature.

If consultants not only command expert knowledge about symptomatic treatments but have sufficient insight to be able to look at the problems of patients and carers, at least in part, through their eyes, an important part of the question posed in the title of this article will already have been answered. However, a further specification is still necessary as to which problems are within the competence of psychiatrists to solve or ameliorate. 'Competence', in this context, includes both professional knowledge and the ability to prioritise and marshal (and continue to monitor and influence) scarce resources into a programme that will promote the long-term welfare of the patient. It also involves the creation and use of information about the progress of the patient and the extent to which key resources are available and actually being utilised. In the mental health team the consultant is often the member best fitted by education and tenure in post to co-ordinate these functions with the key worker.

\section{Audit guidelines}

\section{Clinical audit and needs assessment}

Needs assessment comprises the formulation of a problem profile, prescription of interventions for each problem, skilled staff for each intervention, and settings conducive for therapeutic interaction between health worker and patient. When outcomes are measured at a follow-up assessment, one audit cycle is completed for that individual and another can begin. Audit activities also include peer reviews of cross-sections through this process, either for one patient or for groups with a chosen characteristic in common. The Postgraduate Medical Colleges have accepted that such audit is part of their responsibility for continued medical education.

A start has been made on providing specific consensus guidelines for key issues arising at each of the four levels of appraisal in Box 1, but there is a long way to go. The original Needs For Care Assessment System (Brewin et al, 1987; Brewin \& Wing, 1993) has been updated in a computerised version (details available from CRU). Tools for

Table 1 Health of the Nation Outcome Scales (HoNOS): 12 items and scoring structure
Item
1. Aggression and overactivity $(0-4)$
Subscore
2. Self-harm $(0-4)$
3. Substance misuse $(0-4)$
4. Cognitive dysfunction $(0-4)$
B. Impairment (0-12)
5. Physically ill or disabled (0-4)
6. Hallucinations and delusions $(0-4)$
7. Depression (0-4)
8. Other symptoms (0-4)
9. Social relationships (0-4)
10. Overall level of functioning (0-4)
11. Housing $(0-4)$
12. Occupation (0-4)
A. Behaviour (0-8)

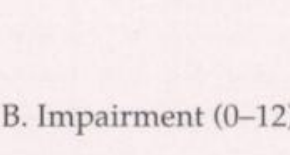
C. Symptoms (0-12)
D. Social problems (0-16)
$\mathrm{A}+\mathrm{B}+\mathrm{C}+\mathrm{D}=0-48$
(Total score) 
Table 2. Auditing district services. Total scores, and subscores for purchasers and providers

$\begin{array}{llll}\text { District } & \begin{array}{l}\text { Provider } \\ \text { 13 points }\end{array} & \begin{array}{l}\text { Purchaser } \\ 7 \text { points }\end{array} & \begin{array}{l}\text { Total } \\ 20 \text { points }\end{array} \\ \text { A } & 37 & 18 & 55 \\ \text { B } & 32 & 21 & 53 \\ \text { C } & 34 & 16 & 50 \\ \text { D } & 31 & 18 & 49 \\ \text { E } & 23 & 18 & 41 \\ \text { F } & 27 & 13 & 40 \\ \text { G } & 23 & 11 & 34 \\ \text { H } & 23 & 10 & 33 \\ \text { I } & 19 & 13 & 32 \\ \text { J } & 12 & 7 & 19 \\ \text { K } & 10 & 8 & 18 \\ \text { Total } & 271 & 153 & 424 \\ \text { Mean } & 20.9 & 21.9 & 21.2 \\ & & & \end{array}$

rating a symptom profile and then allocating a differential diagnosis (including schizophrenia) are also rapidly becoming available, based on the criteria for ICD-10 (WHO, 1992, 1994) and DSMIV (APA, 1994). A brief 12-item instrument called Health of the Nation Outcome Scales (HoNOS) for possible routine use in the NHS, principally by nurses and to a lesser extent by psychiatrists, has been tested on a large scale and in a wide variety of settings. Schizophrenia was the second most common diagnosis in the series. The instrument is robust, acceptable to users, sensitive to change (or lack of it) over time, and has been found reliable in independent trials. It correlates well with scales with a longer track record (details from CRU). Table 1 lists the HoNOS items and shows the scoring structure.

The College has recently promulgated Clinical Practice Guidelines (CPGs) on depression (Paykel \& Priest, 1992), high dose neuroleptics (RCPsych, 1993), and the administration of ECT (Pippard, 1992; Freeman, 1995). A formal development procedure for creating such guidance in full consultation with the membership of the College has now been set up (RCPsych, 1994; Marriott, 1995) and the first product will be CPGs on the management of violence. A useful summary of adverse reactions to antipsychotic drugs has been circulated in Prescribers' Journal (Szabadi, 1995). A recent supplement to European Psychiatry has been devoted to treatment-resistant schizophrenia (Melzer \& Hippius, 1995).

The list of specific and good quality guidance and discussion is already substantial but it has to be supplemented by data on the quality, availability and use of services. The problem of coping with the resulting information overload will be discussed later.

\section{Commissioning and providing services}

The clinical audit approach is based on the care given to individual patients or groups selected for specific characteristics. However, service functions that are vital to clinical quality but are not within the immediate power of the clinician to influence in an individual case, may be missed during routine audit. The Clinical Standards Advisory Group (CSAG), a Government-appointed but clinically-led group responsible for initiating new standards in selected areas of clinical practice, set up a committee to commission a set of guidelines for schizophrenia (CSAG, 1995). The College Research Unit (CRU) searched the literature but found nothing suitable for immediate use or simple adaptation and a set was therefore drafted for the purpose. This was used as a pilot for the initial appraisal stage of an audit cycle. Clinical teams made two day visits to eleven districts; one in each of the eight English Regions, plus one each in Northern Ireland, Scotland and Wales. Both commissioning and providing aspects were covered. Volume one of the subsequent report is highly relevant to the theme of this paper, since it describes and evaluates the experience and working conditions of clinicians, administrators and public health commissioners (CSAG, 1995).

Box 2. Characteristics of high morale districts:

Relations between purchaser and provider executives are characterised by: Informed and sympathetic interaction Shared vision

Commitment to clear aims and achievable targets

The organisation is characterised by: Stability

Management not in constant flux Staff turnover deliberate and progressive

Clinical activity is characterised by: Strong clinical leadership Shared audit and accountability between clinicians and managers

Clinicians involved in decisions about the development of their services Clinicians have reasonable control over the demands of their jobs

Resources are adequate to achieve acceptable standards of care 
On the purchasing side, the teams considered areas such as population needs assessment (Glover et al, 1995), purchasing strategy, service specification, quality assurance, and interaction with other purchasers. On the providing side, the issues included the business plan, ward and community services, crisis management, clinical interventions, social services and the involvement of users and carers.

Overall ratings on a $0-4$ scale of quality (very poor, poor, acceptable, good, excellent) were made on seven key points for commissioners and 13 for providers in each district. Table 2 shows the two mean subtotals and the total score for each of the eleven districts. The two subtotals were highly correlated, suggesting that the total score could be regarded as a useful measure of the extent to which districts were meeting the guidelines. Four districts with the highest mean totals had an average key point score close to 'good'. Five were close to 'acceptable' and the two remaining districts did not quite reach 'poor'. It was clear, however, that even in the last two districts, some of the clinical items scored well - clinicians doing the best they could in spite of the circumstances.

The term 'morale' sums up many of the differences that could be discerned between the three groups of districts. The main elements are listed in Box 2. If these conditions are not met the organisation becomes demoralised and could fall apart. The clinical contribution to planning and providing is crucial. Volume two of the Report contains a revised version of the Protocol, based on the results of the analysis and further wide consultation (copies available from CRU). The key sections for purchasers and providers are listed in Box 3.

Each section contains a number of ratable items, with suggested prompts. In part one for commissioners, for example, the first section (1.1) is concerned with mental health needs assessment for the population covered. It contains three statements or guidelines, the first of which reads: "Mental health needs assessment is based on population indicators for severe mental illness". More detailed prompts are printed on the opposite page of the booklet, with a list of national and local population indicators that should be considered in assessing local need for services, including indices for:

(a) Poverty, unemployment and more general deprivation.

(b) Social isolation, marital status, migration.

(c) Ethnicity.

(d) Standard mortality ratio, age and sex structure.

(e) Other sociodemographic variables.

(f) Geographical factors, service access (e.g. transport), housing.
Part 2 for providers is structured in the same way. The first section (2.1) covers the provision of community services. It contains four statements or guidelines, the first of which (2.1.1) reads: "Access to community services is prioritised according to diagnosis and risk factors." The more detailed prompts for this item on the opposite page include a diagnosis of schizophrenia or severe mental illness (the DoH definition for which is provided) and the following risk factors:

(a) Serious violence.

(b) Serious self-harm.

(c) Serious self-neglect.

Box 3. Protocols for auditing district services

1. Section for commissioners

1.1 Mental health needs assessment

1.2 Purchasing strategy

1.3 Commissioning and the interaction between purchaser and providers

1.4 Service specification and contract for mental health services

1.5 Contract monitoring and requirements for information

1.6 Implementation, quality and audit procedures

1.7 Interactions with other purchasers (e.g. GPFH and LASS)

1.8 General practitioner fundholding

1.9 Local authority social services

2. Section for providers

2.1 Community services

2.2 Rehabilitation and activity

2.3 Clinical interventions

2.4 Violence and self-harm

2.5 Crisis management

2.6 Short-stay hospital care

2.7 Secure longer-term hospital care

2.8 Longer-term NHS and NHS-hostel accommodation

2.9 Other, mostly non-NHS accommodation

2.10 Clinical records

2.11 Local health service audit procedures

2.12 Education of mental health practitioners

2.13 Local community issues

2.14 Primary care liaison

2.15 Social services and other local authority departments

2.16 Involvement of users and carers

2.17 Morale and leadership 
(d) Serious risk of exploitation.

(e) Patient-specific relapse indicators.

The CSAG Protocol is intended to be used as an audit tool, to discover the strong and weak aspects of districts, without any assumption that all of them can be excellent on all points, all the time. Its design also allows application to specific sectors or services. Following such an appraisal, which could be made by public health departments on a population basis if the expertise were available, the intention would be to discuss the results frankly with all those involved, agree a list of priorities for improvement, and repeat the exercise after a reasonable interval in order to 'complete the audit cycle' and start the next one.

The Care Programme Approach (CPA) has been generally welcomed across the mental health professions. Amplified to include guidance for Supervision Registers and the new Power of Supervised Discharge, it provides a basis on which to build a first model for a mental health information system (Glover, 1995).

\section{Information systems and needs}

Current systems for gathering and disseminating information are unpopular with clinicians because they are designed to provide activity data for management and purchasing and do little to support clinical care. The main exception in the UK is primary care. A substantial proportion of general practitioners have a computer on the desk of the consulting room and many use it to record clinical data as well as to cater for the business needs of the practice. Patients can have the opportunity to see what is recorded about them and be offered a printout. The information system is integrated into the clinical and the administrative work of the practice (Lelliott, 1995).

Systems for secondary care lag further behind. At the moment mental health informatics is divided by two apparently distinct perspectives, conveniently described as 'bottom-up' and 'topdown'. But there should be only one health informatics continuum. A comprehensive Mental Health Information System (MHIS) would cover both kinds of collection and use, and the output would be available, within the limits of confidentiality, to all. Such a system would include both statistical and non-numerical information concern- ing local history, topography and communications; sociodemographic indices and epidemiology; local services (agents and settings), their functioning and efficiency; clinical data about the needs of people in contact with services and how far they are being met; and the opinions of local patients and carers. A great advantage to clinicians would be the facility to include help screens providing a comprehensive library of guidelines and definitions for personal choice.

Such systems would provide a basis for national and regional returns, local resource allocation and target setting, population needs and outcome assessment, casemix, care plans, clinical records, and the audit of clinical care. In order to create and maintain the system, high quality clinical information is needed that can only be obtained with the consistent collaboration of clinical teams, who have to be convinced of the practical value to them and to the people they are caring for. They must also be assured that issues of confidentiality and security have been adequately addressed (Andrews \& Morris-Yates, 1994).

If such a system were available, an outline answer to the question in the title could be obtained by referring to a well-stocked database that recorded the details of clinical contacts and care provided (in the present context) to all those with a diagnosis of schizophrenia on the consultant's list. The relevant printout would include the views of patients and carers. In the case of a long history, relevant cross-sectional and longitudinal summaries could be generated very simply. Moreover, help screens could provide answers to many of the questions that arise during the course of a long illness, such as those on welfare benefits referred to earlier. In addition to details about a particular patient or caseload, the database would be able to compare and contrast the results (aggregated and anonymised as appropriate) from other teams or districts, standardised for sociodemographic profile.

The technology for entering, updating and retrieving such data in a wide variety of formats is already available, but methods of structuring information so that it can routinely be used for selfaudit and group audit has only recently begun to receive practical attention. However, the wellaccepted principles of clinical audit, as amplified and quantified in needs assessment systems informed by consensus guidelines for good practice, can readily be adapted for such use. Useful prototypes are already available (Lelliott et al, 1993). 


\section{Conclusions}

There are two main ways of discovering how well one's clinical performance matches standards. The first is to consult the relevant patients and carers about the problems arising from positive and negative symptoms, both specific and general. In order to maximise the usefulness of the answers (since numerous studies have demonstrated that 'satisfied' answers often hide dissatisfaction) it is essential to be able to see from the same aspect of those experiencing schizophrenia 'from within' or living with it in the family. It is possible to do this to some extent without actually being in the situation oneself. Seen from the two perspectives the problems are different but overlapping and to some extent complementary. Local voluntary organisations can be very helpful and should be supported.

The second way depends on professional knowledge and technical devices for making it readily available for personal, professional and administrative audit. A very simple, but often crucial, example is welfare benefit. Few members of a community team have the information at their fingertips, but they could have if it could readily be brought to a computer screen when needed. Clinical and service data should be similarly available. Much of the requisite knowledge is now potentially available; the problem is one of putting it into an accessible format.

\section{References}

American Psychiatric Association (1994) Diagnostic and Statistical Manual of Mental Disorders (4th edn) (DSM-IV). Washington, DC: APA.

Andrews, G. \& Morris-Yates, A. (1994) Ethical implications of the electronic storage of the medical record. Computers in Mental Health, 1, 129-135.

Brewin, C. R., Wing, J. K., Mangan, S., et al (1987) Principles and practice of measuring needs in the long-term mentally ill. III. The MRC Needs for Care Assessment. Psychological Medicine, 17, 971-981.
— \& - (1993) The MRC Needs for Care Assessment. Progress and controversies. Psychological Medicine, 23, 837-841.

Clinical Standards Advisory Group (1995) Schizophrenia. Report of a CSAG Committee. Volume 1, Report; Volume 2, Protocol. Chairman: A. Sims. London: HMSO.

Creer, C. \& Wing, J. K. (1974) Schizophrenia at Home. Kingstonupon-Thames: National Schizophrenia Fellowship.

Freeman, C. T. (1995) The ECT Handbook. The Second Report of the Royal College of Psychiatrists' Special Committee on ECT. Council Report CR39. London: Royal College of Psychiatrists.

Glover, G. (1995) The public health perspective. In Measurement for Mental Health. Contributions from the College Research Unit. (ed J. K. Wing), pp. 71-80. London: Royal College of Psychiatrists' Research Unit.

- Robin, L., Emami, J., et al (1995) The Mental Illness Needs Index. Crown copyright disc for free use within the UK NHS. Obtainable at cost from RCPsych Research Unit.

Kuipers, L. (1994) The measurement of expressed emotion. International Review of Psychiatry, 6, 187-199.

Lelliott, P. (1995) Mental health information systems: problems and opportunities. Advances in Psychiatric Treatment, 1, 216223.

-, Flannigan, C. \& Shanks, S. (1993) A Review of Seven Mental Health Information Systems. A Functional Perspective. CRU Publication No. 1. London: RCPsych Research Unit.

Marriott, S. (1995) The development of clinical practice guidelines. In Measurement for Mental Health. Contributions from the College Research Unit (ed J. K. Wing), pp. 17-32. London: RCPsych Research Unit.

Melzer, H. Y. \& Hippius, H. (1995) The assessment and management of treatment-resistant schizophrenia in perspective. European Psychiatry, 10, 3-6.

Paykel, E. F. \& Priest, R. G. (1992) Recognition and management of depression in general practice. Consensus statement. British Medical Journal, 305, 1198-1202.

Pippard, J. (1992) Audit of electroconvulsive treatment in two NHS Regions. British Journal of Psychiatry, 160, 621-637.

Royal College of Psychiatrists (1993) Consensus Statement on the use of High Dose Anti-psychotic Medication. Council Report CR26. London: RCPsych.

- (1994) Clinical Practice Guidelines and their Development. Council Report CR34. London: RCPsych.

Sellwood, W., Haddock, G., Tarrier, N., et al (1994) Advances in the psychological management of positive symptoms of schizophrenia. International Review of Psychiatry, 6, 201-215.

Slade, M., McCrone, P. \& Thornicroft, G. (1995) Uptake of welfare benefits by psychiatric patients. Psychiatric Bulletin, 19, 411-413.

Szabadi, E. (1995) Adverse reactions profile: 11. Antipsychotic drugs. Prescribers' Journal, 35, 37-44.

Torry, E. F. (1995) Surviving Schizophrenia. A Family Manual (3rd edn). Harper \& Row.

Wing, J. K. (ed) (1975) Schizophrenia from Within. Kingston-uponThames: National Schizophrenia Fellowship.

World Health Organization (1992) The ICD-10 Classification of Mental and Behavioural Disorders. Conversion Tables between ICD-8, ICD-9 and ICD-10. Geneva: WHO.

- (1994) SCAN. Schedules for Clinical Assessment in Neuropsychiatry. Geneva: WHO. 